\title{
Necessidades não atendidas de cuidados cirúrgicos para crianças: Estudo de caso no Sistema Único de Saúde financiado pelo governo no Brasil ${ }^{*}$
}

\section{Unmet Needs of Surgical Care for Children: A Case Study in the Brazilian Publicly-Financed Health System}

\author{
Mario Bressan-Neto ${ }^{1}$ (1) Marina Rosa Filezio ${ }^{20}$ \\ ${ }^{1}$ Departamento de Ortopedia e Anestesiologia, Faculdade de \\ Medicina de Ribeirão Preto, Universidade de São Paulo, Ribeirão \\ Preto, São Paulo, Brasil \\ ${ }^{2}$ Divisão de Ortopedia Mecânica Pediátrica, Cumming School of \\ Medicine, University of Calgary, Calgary, Alberta, Canadá \\ ${ }^{3}$ Departamento de Biomecânica, Medicina e Reabilitação, Faculdade \\ de Medicina de Ribeirão Preto, Universidade de São Paulo, Ribeirão \\ Preto, São Paulo, Brasil
}

Fabio Ferri-de-Barros ${ }^{2}$

Helton Luiz Aparecido Defino 3 (1)

Endereço para correspondência Mario Bressan-Neto, MSc, Departamento de Ortopedia e Anestesiologia, Faculdade de Medicina de Ribeirão Preto, Universidade de São Paulo, Avenida dos Bandeirantes, $3.900,11^{\circ}$ andar, Ribeirão Preto, SP, 14048-900, Brasil (e-mail: mbressan@hotmail.com).

Rev Bras Ortop 2021;56(3):360-367.

\begin{abstract}
Resumo
Palavras-chave

- coluna

- escoliose

- listas de espera

- curvaturas vertebrais

- política de saúde

Objetivo Medir e documentar o impacto clínico do tempo de espera para tratamento cirúrgico de pacientes com deformidades na coluna vertebral em um centro quaternário no Brasil.

Métodos No total, 59 pacientes com deformidade espinhal à espera de cirurgia na lista do nosso hospital foram avaliados para observar o impacto dos tempos de espera na progressão da deformidade. A avaliação do paciente foi realizada utilizando o questionário SRS-22r para qualidade de vida relacionada à saúde (QLRS), e imagens radiográficas para avaliar a deformidade da coluna vertebral quando os pacientes foram incluídos na lista de espera e na consulta mais recente. Os parâmetros radiográficos selecionados para comparação foram: ângulo de Cobb de curvas primárias e secundárias, alinhamento coronal, translação de vértebra apical, obliquidade pélvica, eixo vertebral sagital, cifose (T5-T12), e lordose (L1-S1). Resultados Baixos escores de QLRS segundo o questionário SRS-22r foram observados em pacientes que aguardavam cirurgia. Os parâmetros radiográficos mostraram progressão da deformidade na avaliação inicial em comparação com a avaliação de seguimento mais recente.

Conclusão Os pacientes que aguardavam tratamento cirúrgico de deformidade espinhal em nosso centro apresentaram os escores de QLRS relativamente baixos e progressão radiográfica da deformidade.
\end{abstract}

Trabalho desenvolvido no Departamento de Biomecânica, Medicina e Reabilitação, Faculdade de Medicina de Ribeirão Preto, Universidade de São Paulo, Ribeirão Preto, São Paulo, Brasil. recebido

27 de Maio de 2020

aceito

16 de Setembro de 2020

Publicado on-line

Março 30, 2021
DOI https://doi.org/ $10.1055 / \mathrm{s}-0040-1721836$ ISSN $0102-3616$.
(C) 2021. Sociedade Brasileira de Ortopedia e Traumatologia. All rights reserved.

This is an open access article published by Thieme under the terms of the Creative Commons Attribution-NonDerivative-NonCommercial-License, permitting copying and reproduction so long as the original work is given appropriate credit. Contents may not be used for commercial purposes, or adapted, remixed, transformed or built upon. (https://creativecommons.org/ licenses/by-nc-nd/4.0/)

Thieme Revinter Publicações Ltda., Rua do Matoso 170, Rio de Janeiro, RJ, CEP 20270-135, Brazil 
Abstract

Keywords
- spine
- scoliosis
- waiting list
- vertebral curvatures
- health policy

Objective To measure and document the clinical impact of the waiting time for surgical treatment of patients with spinal deformities in a quaternary center in Brazil. Methods In total, 59 patients with spinal deformity waiting for surgery on our hospital's list were evaluated to observe the impact of the waiting time on the progression of the deformity. Patient evaluation was performed using the SRS-22r questionnaire for health-related quality of life (HRQL) and radiographic images to evaluate the deformity of the spine at the time the patients were included in the waiting list and at the most recent appointment. The radiographic parameters selected for comparison were: Cobb angle of the primary and secondary curves, coronal alignment, apical vertebral translation, pelvic obliquity, sagittal vertebral axis, kyphosis (T5-T12), and lordosis (L1-S1).

Results Low HRQL scores according to the SRS-22r questionnaire were observed in patients waiting for surgery. The radiographic parameters showed progression of the deformity on the initial evaluation when compared with the most recent follow-up evaluation.

Conclusion The patients waiting for surgical treatment of spinal deformities in our center showed relatively low HRQL scores and radiographic progression of the deformity.

\section{Introdução}

A organização do Sistema Único de Saúde (SUS) determina que o tratamento cirúrgico das deformidades da coluna vertebral deve ser realizado em centros terciários especializados. ${ }^{1}$ Os pacientes encaminhados para centros terciários entram em fila de espera.

O tratamento cirúrgico das deformidades da coluna vertebral tem características especiais (longa duração das cirurgias, exigência de recursos humanos especializados, alto custo de implantes e recursos técnicos). Isso, associado ao subfinanciamento do SUS, tem levado a um aumento constante na lista de espera cirúrgica. ${ }^{2,3}$

Evidências emergentes sugerem que o tratamento para escoliose é sensível ao tempo, pois a escoliose piora com o crescimento da coluna vertebral e com o passar do tempo. ${ }^{4-7}$ À medida que os pacientes aguardam tratamento, particularmente crianças e jovens, suas deformidades na coluna se deterioram, tornando-se mais complexas e mórbidas, causando sofrimento emocional indevido para os pacientes e seus familiares. ${ }^{8}$ Além disso, o risco de complicações do tratamento cirúrgico de deformidades espinhais maiores é substancialmente mais alto, ${ }^{9}$ assim como o custo do tratamento. ${ }^{10,11}$ Alguns estudos mostraram o impacto dos longos tempos de espera para o tratamento cirúrgico da escoliose no Canadá ${ }^{4,7,12}$ e no Brasil. 3,13,14

Apesar da melhora na atenção primária à saúde, o SUS tem enfrentado desafios na prestação de cuidados de saúde universais e equitativos a 209 milhões de brasileiros. ${ }^{15}$ As decisões de alocação e o planejamento ocorrem nas Conferências Nacionais de Saúde, que são realizadas a cada quatro anos, de acordo com uma lei federal. ${ }^{16} \mathrm{O}$ atual processo de tomada de decisão para a alocação de recursos em saúde para o SUS tem sistematicamente falhado em responder às necessidades não atendidas de cuidados cirúrgicos de crianças e jovens que são desproporcionalmente sobrecarregados com a falta de acesso à assistência hospitalar no Brasil. ${ }^{17-19}$

Em um dos maiores hospitais acadêmicos quaternários do Brasil, um dos autores seniores notou nos últimos dez anos um impacto dramático da crescente carga de escoliose em decorrência da atual política pública de saúde, ou da falta dela, para a alocação de recursos cirúrgicos para crianças e jovens com deformidades espinhais. O objetivo deste estudo de caso é medir e documentar o impacto clínico do tempo de espera para tratamento cirúrgico de pacientes com deformidades complexas da coluna vertebral em um centro quaternário no Brasil.

\section{Materiais e Métodos}

Esta série de casos retrospectivos foi aprovada pelo comitê de ética em pesquisa sob o número 833.475. Foi avaliada uma coorte de 59 pacientes com deformidades na coluna vertebral na lista de espera cirúrgica a partir de dezembro de 2013 em um centro quaternário no Brasil. Apenas deformidades pediátricas, definidas pela idade e etiologia do diagnóstico, foram consideradas no estudo. Foram excluídas deformidades adultas ou degenerativas, bem como um paciente que estava na lista de espera e já havia sido submetido a cirurgia em outro hospital.

Os prontuários dos pacientes e as radiografias da coluna vertebral foram revisados. As principais medidas de desfecho incluíram tempo de espera para cirurgia (quanto tempo os pacientes aguardaram pelo tratamento cirúrgico até dezembro de 2013) e qualidade de vida relacionada à saúde (QLRS) utilizando o questionário SRS-22r validado em português. ${ }^{20}$ O questionário foi aplicado para pacientes com mais de 10 anos de idade e com função cognitiva completa. 
As imagens radiográficas foram avaliadas quando o tratamento cirúrgico foi recomendado (inclusão na lista de espera) e na consulta de acompanhamento mais recente. As medições radiográficas foram feitas manualmente em imagens radiográficas impressas e digitais, ${ }^{21}$ utilizando o programa Osirix (Pixmeo Sarl, Bernex, Suíça). Os parâmetros radiográficos selecionados para comparação foram: ângulo de Cobb de curvas primárias e secundárias, alinhamento coronal, translação de vértebra apical, obliquidade pélvica, eixo vertebral sagital, cifose (T5-T12), e lordose (L1-S1). Para pacientes com escoliose neuromuscular, a obliquidade pélvica foi avaliada de acordo com Gupta et al. ${ }^{22}$

Analisamos os dados com o programa John's Macintosh Project (JMP, SAS Institute, Inc., Cary, Carolina do Norte, EUA). Usamos o teste $t$ de Student para médias e desvios-padrão para dados dados de distribuição normal. Para dados com distribuição não paramétrica, calculamos medianas e intervalos interquartil (IIQs), que analisamos com análise de variância (analysis of variance, ANOVA, em inglês) e o teste U de Mann-Whitney (análise intergrupo). Testes $t$ de Student emparelhados foram utilizados para a análise intragrupo. A análise correspondente foi descrita com a diferença média e o intervalo de confiança de 95\% (IC95\%). O nível de significância $(\alpha)$ foi estabelecido como 0,05 .

\section{Resultados}

Foram avaliados 59 pacientes (40 mulheres) que estavam na lista de espera cirúrgica para tratamento de deformidades na coluna vertebral em 31 de dezembro de 2013. A idade dos pacientes variou de 3 a 23 anos (média de 13,5 \pm 3,7 anos). A etiologia das deformidades foi: neuromuscular (17 pacientes; $28,3 \%$ ), congênita (16 pacientes; $26,7 \%$ ), idiopática (15 pacientes; $25,0 \%$ ), sindrômica (10 pacientes; $16,7 \%$ ), síndrome de Marfan (1 paciente; $1,7 \%$ ), e neurofibromatose (1 paciente; $1,7 \%$ ). 0 tempo de espera para a cirurgia em dezembro de 2013 variou de 2 a 117 meses (mediana: 13,5 meses; IIQ: 13,8 meses).

A avaliação da QLRS foi feita com o questionário SRS-22r em 36 pacientes com as seguintes etiologias: 11 (30,6\%) - neuromuscular,;10 (27,8\%) - idiopática; 8 (22,2\%) - congênita; 5 (13,9\%) - sindrômica; 1 (2,8\%) - síndrome de Marfan; e 1 (2,8\%) - neurofibromatose. A pontuação mediana para cada categoria foi: função - 3,60 (IIQ: 1,00); dor - 4,00 (IIQ: 1,40); autoimagem - 3,00 (IIQ: 0,80); saúde mental - 3,80 (IIQ: 1,00); e satisfação - 4,00 (IIQ: 1,00) (-Fig. 1).

Os parâmetros radiográficos apresentaram diferenças estatisticamente significativas comparando a avaliação no momento da indicação cirúrgica e no seguimento. Observouse diferença estatística nos parâmetros coronal e sagital que indicam a progressão da deformidade ( - Tabela 1, - Figs. 1, 2, 3 e 4). Entre os pacientes com esqueletos imaturos na avaliação inicial, $18(58,1 \%)$ atingiram a maturidade esquelética enquanto aguardavam a cirurgia.

No plano coronal, o ângulo de Cobb da deformidade principal aumentou em média $18,6^{\circ}$ (IC95\%: $13,9^{\circ}$ a $23,4^{\circ}$; $p<0,0001$ ). 0 aumento da deformidade foi observado em todas as etiologias (-Fig. 5). O ângulo de Cobb da curva

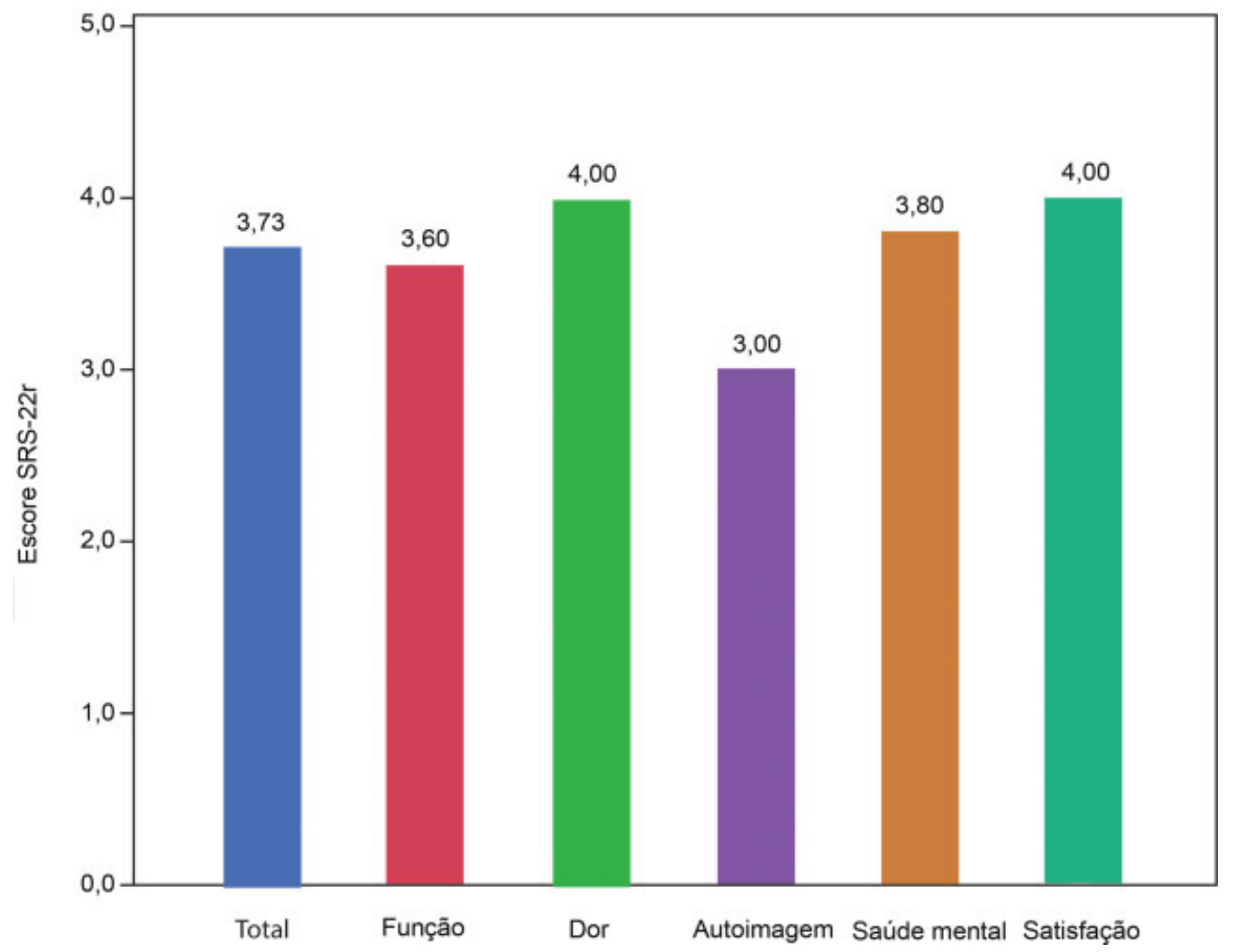

Fig. 1 Resultado dos pacientes na lista de espera cirúrgica de acordo com os escores do questionário SRS-22r. Cada barra corresponde à pontuação média de cada domínio no questionário. 
Tabela 1 Resumo dos parâmetros radiográficos nas avaliações inicial e final, diferença média, intervalo de confiança de 95\% (IC 95\%) e valor de $p$ para análise emparelhada

\begin{tabular}{|l|l|l|l|l|l|}
\hline Parâmetros radiográficos & Inicial & Final & Diferença média & IC95\% & Valor de $p$ \\
\hline Plano coronal & & & & & \\
\hline Curva principal & $61,19^{\circ}$ & $79,81^{\circ}$ & $18,61^{\circ}$ & $13,88^{\circ}-23,35^{\circ}$ & $<0,0001^{*}$ \\
\hline Curva secundária 1 & $39,07^{\circ}$ & $49,73^{\circ}$ & $10,66^{\circ}$ & $7,73^{\circ}-13,58^{\circ}$ & $<0,0001^{*}$ \\
\hline Curva secundária 2 & $21,16^{\circ}$ & $26,78^{\circ}$ & $5,63^{\circ}$ & $1,54^{\circ}-9,72^{\circ}$ & $0,0086^{*}$ \\
\hline C7-CSVL (milímetros) & 21,54 & 31,73 & 10,20 & $2,44-17,95$ & $0,0113^{*}$ \\
\hline AVT (milímetros) & 38,56 & 55,85 & 17,28 & $8,6-25,97$ & $0,0003^{*}$ \\
\hline Obliquidade pélvica (horizontal) & $8,88^{\circ}$ & $12,68^{\circ}$ & $3,80^{\circ}$ & $0,43^{\circ}-7,17^{\circ}$ & $0,0287^{*}$ \\
\hline Obliquidade pélvica (T1) & $12,44^{\circ}$ & $16,48^{\circ}$ & $4,04^{\circ}$ & $0,27^{\circ}-7,81^{\circ}$ & $0,0369^{*}$ \\
\hline Plano sagital & & & & $1,55-21,39$ & $0,0245^{*}$ \\
\hline EVS (milímetros) & 29,53 & 41,00 & 11,47 & $-0,04^{\circ}-11,80^{\circ}$ & 0,05 \\
\hline Cifose (T5-T12) & $33,74^{\circ}$ & $39,62^{\circ}$ & $5,88^{\circ}$ & $-6,59^{\circ}-6,09^{\circ}$ & 0,937 \\
\hline Lordose (L1-S1) & $-54,80^{\circ}$ & $-57,05^{\circ}$ & $-0,25^{\circ}$ & $10,51^{\circ}-27,03^{\circ}$ & $0,0003^{*}$ \\
\hline Principal deformidade sagital & $69,15^{\circ}$ & $87,92^{\circ}$ & $18,77^{\circ}$ & & \\
\hline
\end{tabular}

Abreviaturas: SVA, eixo vertical sagital; IC95\%, intervalo de confiança de 95\%; C7-CSVL, linha vertical sacral central; AVT, translação da vértebra apical; Nota: *significância estatística.
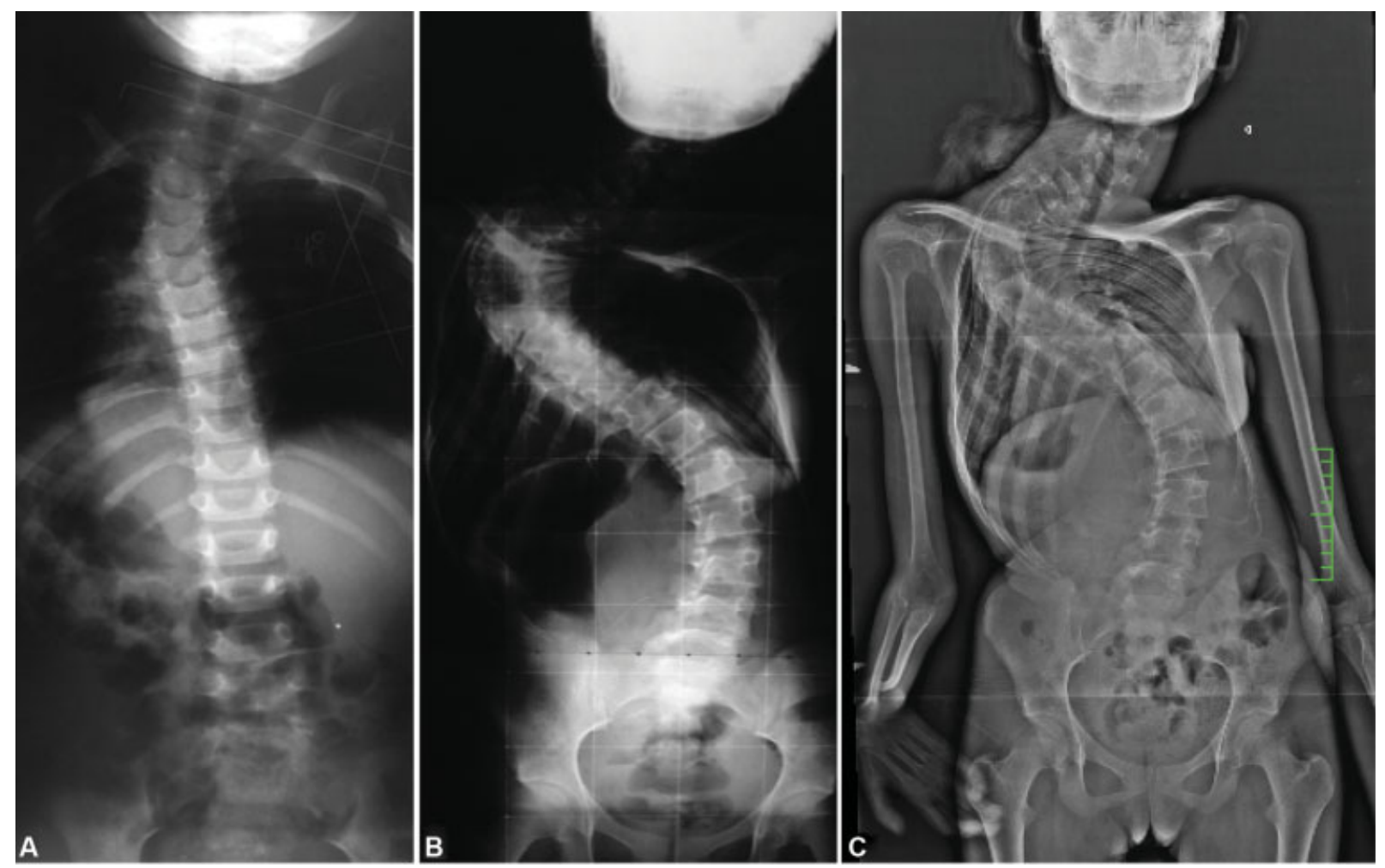

Fig. 2 Progressão radiográfica da escoliose idiopática adolescente a partir de 2005 (A), em 2010 (B), e em 2013 (C).

secundária aumentou em média $10,7^{\circ}$ (IC95\%: $7,7^{\circ}$ a $13,6^{\circ}$; $p<0,0001$ ) (-Fig. 6).

\section{Discussão}

Este estudo documenta o impacto do longo tempo de espera para o tratamento cirúrgico de deformidades da coluna vertebral em crianças e jovens em um centro quaternário no Sistema Único de Saúde (SUS). A avaliação dos pacientes em nossa lista de espera para tratamento cirúrgico de deformidades espinhais mostrou progressão das deformidades e diminuição dos escores de QLRS. Contestamos o termo "esperar por cirurgia" porque muitos pacientes nunca foram operados até o momento. A realização de tratamento 
364 Necessidades não atendidas de cuidados cirúrgicos para crianças Bressan-Neto et al.

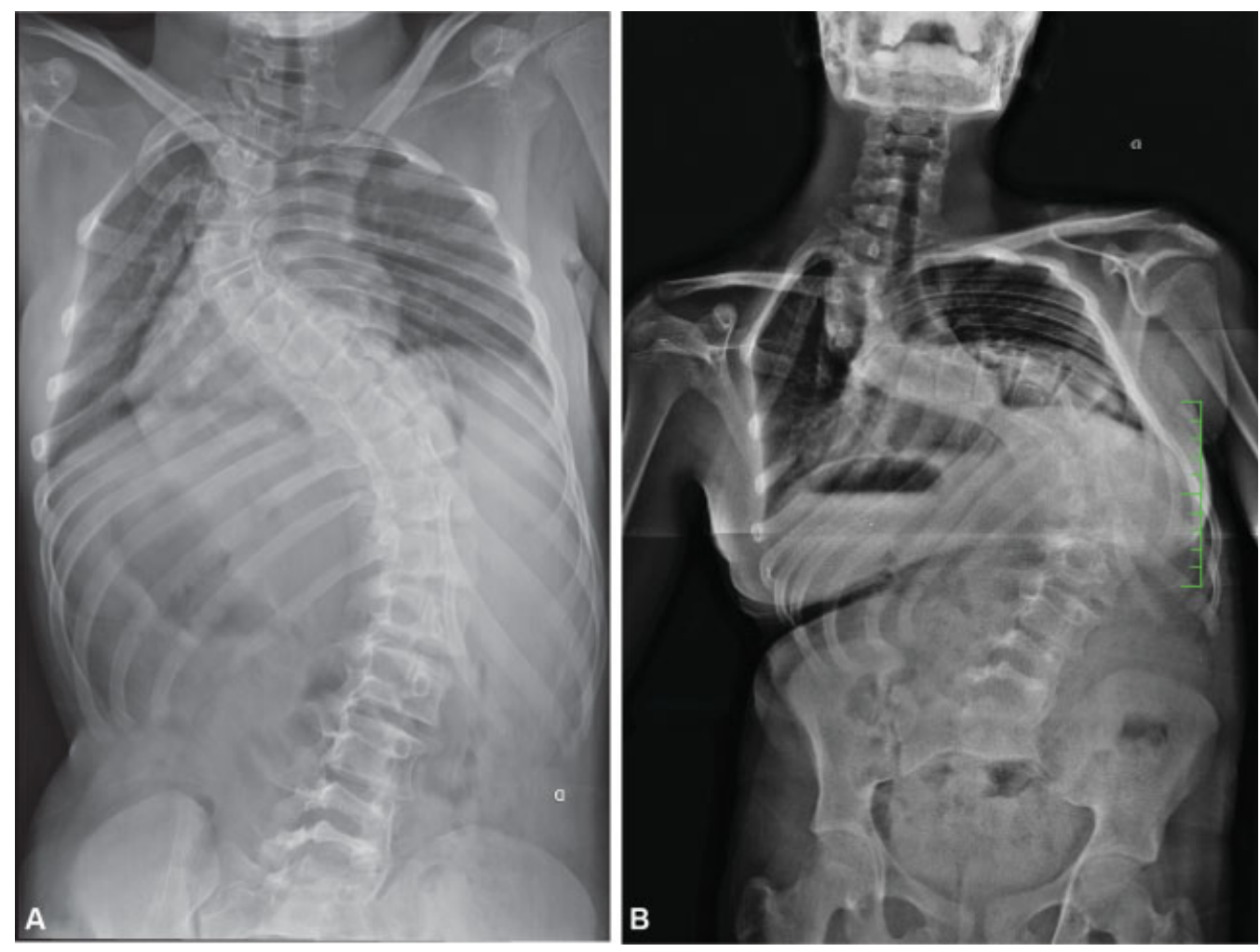

Fig. 3 Progressão da escoliose sindrômica em uma menina de 10 anos de fevereiro de 2013 (A) a janeiro de 2014 (B).

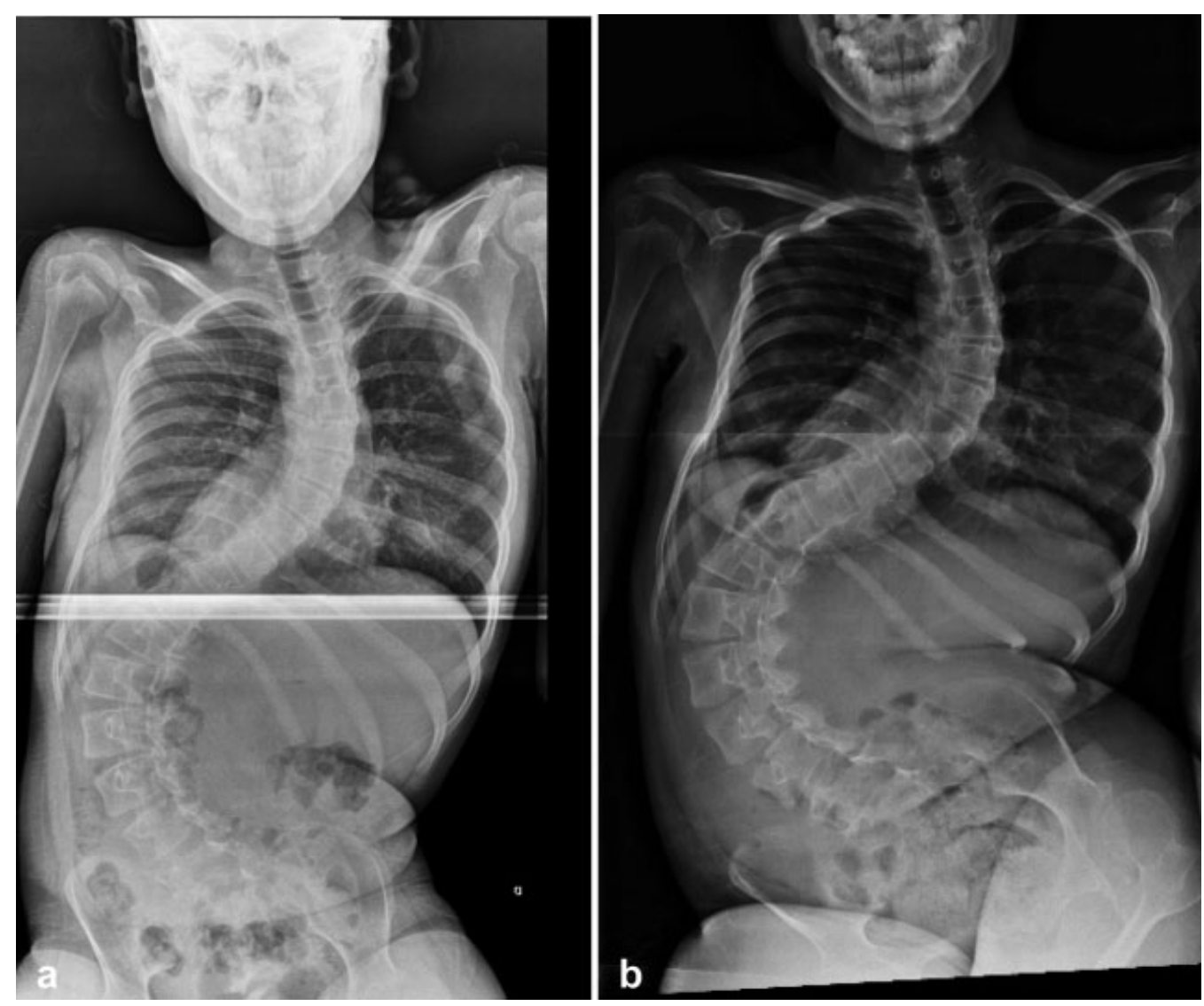

Fig. 4 Progressão de deformidade de janeiro (A) a outubro de 2013 (B) em paciente com amiotrofia espinhal. 


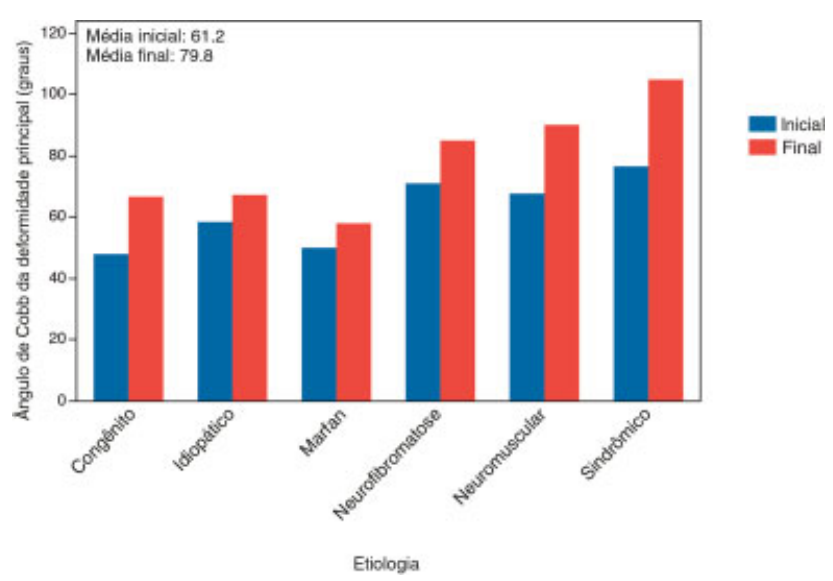

Fig. 5 Comparação entre os ângulos inicial e final de Cobb da curva principal de deformidade nas diferentes etiologias, mostrando piora da deformidade em todos os subgrupos de pacientes.

cirúrgico para maiores deformidades vertebrais, à medida que elas avançam com o tempo, representa aumento do custo e da morbidade, e, em alguns casos extremos, o alto risco de complicações fatais pode impedir que os cirurgiões realizem o tratamento cirúrgico recomendado. 0 crescente número de processos judiciais para tratamento médico hospitalar no Brasil $^{23}$ ilustra esse complexo problema da política de saúde e os desafios envolvidos na incorporação de tecnologia e tratamentos complexos (e seus custos inerentes) em um sistema de saúde com recursos financeiros limitados. 24

Os pacientes na lista de espera do nosso estudo foram manejados de acordo com as políticas de saúde atuais do SUS; porém, essa abordagem não tem sido eficaz, como mostram nossos dados. O SUS foi criado após a Constituição brasileira de 1988 reconhecer a saúde como direito e dever do Estado. ${ }^{15,16}$ Problemas relacionados ao tempo de espera para o tratamento de deformidades da coluna vertebral foram relatados no Brasil ${ }^{3,13,14}$ e em outros países como Canadá, Reino Unido, ${ }^{10}$ e Nova Zelândia. ${ }^{8} \mathrm{O}$ tempo médio de espera foi de 1 ano no Canadá, , 7,12 de 5 a 9 meses no Reino Unido (segundo Clark $^{10}$ ), e de 2,5 semanas a 2,9 anos na Nova Zelândia. ${ }^{8}$ Os perigos dos tempos de espera prolongados são muito conhecidos e caracterizados pela progressão da curva, pelo aumento dos sintomas, e pelo impacto negativo na saúde mental e na qualidade de vida do paciente. ${ }^{6,7,25}$ Os resultados observados em nosso estudo apenas corroboram e concordam com os relatórios anteriores.

Ao estudar a QLRS dos pacientes na lista de espera, pudemos observar baixos escores no questionário específico de QLRS para pacientes com deformidades espinhais (SRS-22r). Assim, Calman et al. ${ }^{8}$ avaliaram o impacto do atraso no tratamento cirúrgico para pacientes com escoliose idiopática correlacionado com o agravamento progressivo da QLRS. Em nosso estudo, quando a decisão cirúrgica foi tomada, não havia dados

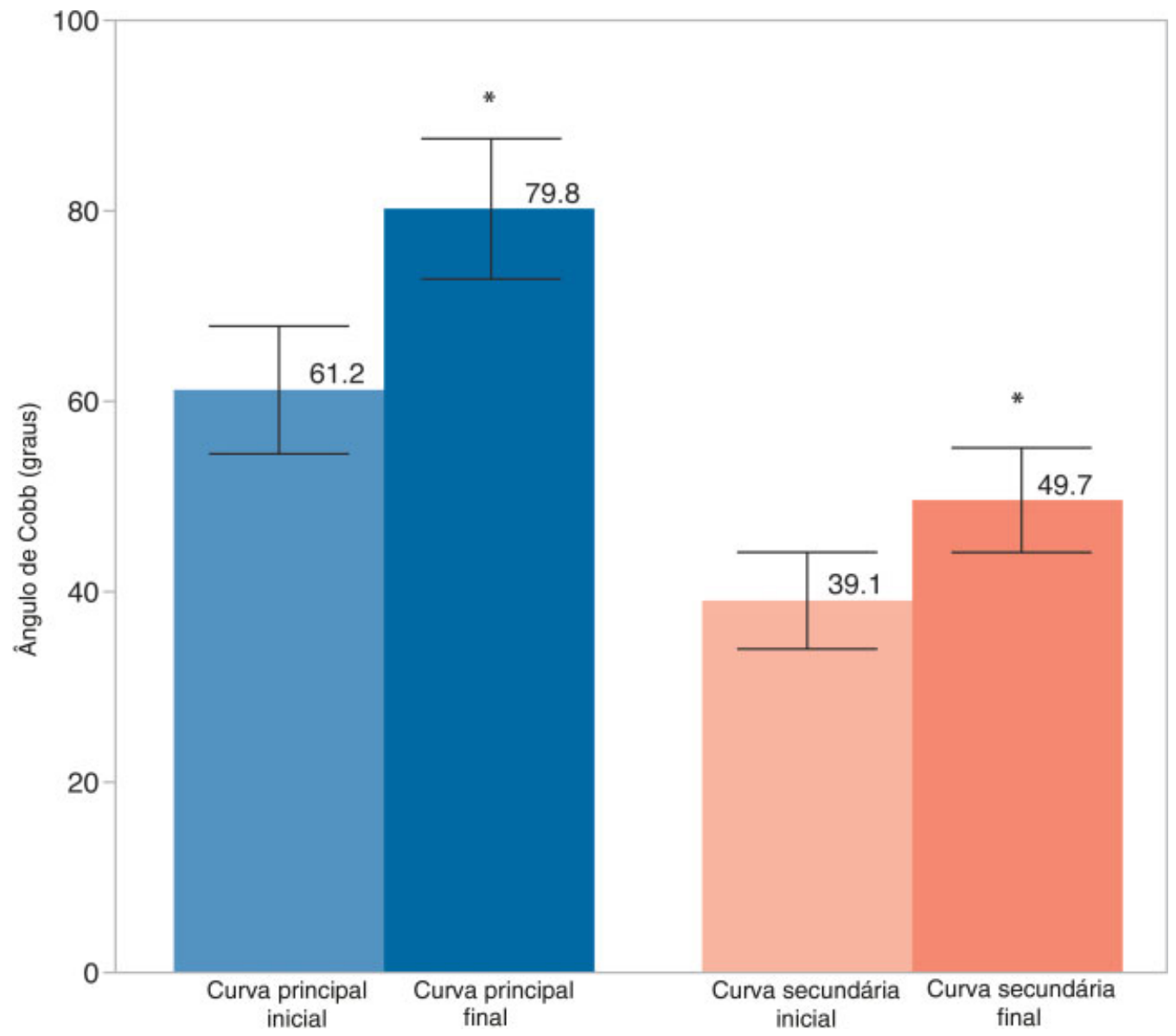

Fig. 6 Comparação entre os ângulos inicial e final de Cobb das curvas principal e secundária. As barras e os números representam o ângulo médio de Cobb, e as barras de erro representam o IC95\%. O asterisco $\left(^{*}\right)$ indica diferença estatística. 
Tabela 2 Comparação entre pontuação do questionário SRS-22r e dados da literatura

\begin{tabular}{|l|l|l|l|}
\hline Domínio (SRS-22r) & Lista de espera cirúrgica (avaliação clínica) & Escoliose idiopática $^{20}$ & Escoliose congênita $^{26}$ \\
\hline Função & $3,60(1,00)$ & $4,08 \pm 0,75$ & $4,64 \pm 0,5$ \\
\hline Dor & $4,00(1,40)$ & $3,99 \pm 0,87$ & $4,53 \pm 0,47$ \\
\hline Autoimagem & $3,00(0,80)$ & $3,53 \pm 0,83$ & $3,73 \pm 0,85$ \\
\hline Saúde mental & $3,80(1,00)$ & $3,73 \pm 0,75$ & $4,21 \pm 0,59$ \\
\hline Satisfação & $4,00(1,00)$ & $4,28 \pm 0,83$ & $4,02 \pm 0,88$ \\
\hline Total & $3,73(0,91)$ & Indisponível & $4,23 \pm 0,52$ \\
\hline
\end{tabular}

Notas: ${ }^{\dagger}$ dados expressos na faixa mediana e interquartil (entre parênteses); *dados expressos em desvio médio e padrão, adaptado de Camarini et al. ${ }^{20}$ e de Farley et al. ${ }^{26}$

de QLRS de linha de base, e isso é uma limitação. Independente disso, pudemos observar pontuações no SRS-22 menores do que as descritas na literatura. Camarini et al., ${ }^{20}$ no estudo que resultou na validação do SRS-22r para a população brasileira, aplicaram o questionário a pacientes com escoliose idiopática e obtiveram pontuação superior à do nosso estudo, exceto nas categorias "dor" e "saúde mental", que eram as mesmas do que a nossa. Farley et al. ${ }^{26}$ aplicaram o questionário SRS-22r a pacientes com escoliose congênita, e obtiveram escores maiores em cada categoria (- Tabela $\mathbf{2}$ ).

A avaliação das consequências radiográficas mostrou agravamento das deformidades do paciente enquanto aguardava o procedimento cirúrgico. Houve aumento nos ângulos das deformidades primárias e secundárias, progressão do desequilíbrio nos planos coronal e sagital, e aumento do número de pacientes com obliquidade pélvica. Assim, Dabke et al. ${ }^{6}$ realizara uma análise retrospectiva em pacientes adolescentes com escoliose idiopática tratada cirurgicamente, e relatou piora significativa da deformidade enquanto os pacientes aguardavam a cirurgia, o que resultou em cirurgias mais complexas a serem realizadas do que as originalmente previstas em 16,7\% dos casos. Miyanji et al. ${ }^{9}$ revisaram o tratamento de 325 pacientes com escoliose idiopática, e correlacionaram o aumento da deformidade com o tempo cirúrgico, o número de níveis incluídos na cirurgia, e o risco de necessidade de transfusão sanguínea. Embora o estudo não incluísse a análise dos custos cirúrgicos, os autores concluíram que o aumento da utilização dos recursos resulta em um aumento das despesas com o tratamento. Em outro estudo, Miyanji et al. ${ }^{7}$ analisaram a perspectiva dos cirurgiões responsáveis para o tratamento de pacientes com deformidade espinhal, e relataram que o aumento da gravidade da deformidade enquanto os pacientes aguardam o procedimento cirúrgico leva os cirurgiões a planejar um procedimento mais difícil e mórbido. Ou seja, segundo a literatura, a piora radiográfica observada em nosso estudo significa procedimentos mais complexos, com consequências clínicas para os pacientes e consequências financeiras para o sistema de saúde. Em nosso estudo, além do aumento da gravidade da deformidade e o consequente desequilíbrio observado enquanto os pacientes aguardam tratamento cirúrgico, também houve aumento no número de pacientes com obliquidade pélvica que necessitava de instrumentação pélvica. A inclusão da pélvis leva a um aumento no tempo cirúrgico, perda de sangue, e risco de infecção. ${ }^{27-30}$ Martin et al. ${ }^{31}$ analisaram um banco de dados multicêntrico com 1.890 pacientes submetidos a cirurgia por deformidade pediátrica da coluna vertebral, e identificaram um aumento na complexidade do procedimento, particularmente em pacientes que incluíram fixação pélvica, como fator de risco para reinternação hospitalar não planejada nos primeiros trinta dias após a operação. Com os resultados de maiores riscos de complicações, reinternação hospitalar, e maiores custos de implante pélvico, os autores concluíram que pacientes com obliquidade pélvica precisarão de cirurgias mais caras.

Listas de espera são comuns a todos os serviços financiados publicamente em todo o mundo. ${ }^{25,32}$ Longos tempos de espera para o tratamento cirúrgico têm corroído a confiança dos cidadãos no sistema de saúde. ${ }^{33}$ Portanto, os tempos de espera cirúrgicos tornaram-se uma importante questão social e política. O impacto negativo dos tempos de espera prolongados para a cirurgia de deformidade da coluna vertebral foi reconhecido. Foram feitas tentativas de estabelecer um tempo de espera máximo aceitável com base na minimização do risco de cirurgia adicional devido à progressão de deformidade. Como exemplo, o Canadian Pediatric Surgical Times Project propôs um tempo máximo de espera de seis meses com base na opinião de um especialista, que foi contestado e revisado para três meses com base em dados empíricos. ${ }^{4}$

Em nosso estudo, avaliamos todos os pacientes que aguardavam a correção cirúrgica de sua deformidade, e não apenas os pacientes que receberam o tratamento. Reconhecemos que o tempo de espera para a cirurgia e as consequências desse atraso podem ser subestimados. No entanto, alguns desses pacientes podem nunca receber o tratamento desejado, e, caso contrário, não seriam reconhecidos. Nosso estudo se soma à literatura que exige uma melhora das políticas de saúde para dar conta das necessidades não atendidas do cuidado cirúrgico a crianças e jovens brasileiros. Mais pesquisas sobre o tema são necessárias para facilitar a formulação de políticas de saúde baseadas em evidências no Brasil.

\section{Conclusão}

Neste estudo, com o tempo médio de espera de 13 meses para o tratamento cirúrgico de deformidades da coluna vertebral de diversas etiologias, documentamos o agravamento das 
deformidades e a deterioração da QLRS, o que está de acordo com estudos anteriores. Isso representa um aumento evitável na carga da doença e no custo do tratamento. A política de saúde pública para o manejo de pacientes com deformidades na coluna vertebral no Brasil deve visar melhorar o acesso à assistência cirúrgica de crianças e jovens para mitigar essa fardo evitável.

\section{Suporte Financeiro}

Não houve suporte financeiro de fontes públicas, comerciais, ou sem fins lucrativos.

\section{Conflito de Interesses}

Os autores declaram não haver conflito de interesses.

\section{Referências}

1 Brasil. Conselho Nacional de Secretários de Saúde. Assistência de média e alta complexidade no SUS. Brasília2011. (Coleção para entender a gestão do SUS 2011, v.4)

2 Bressan-Neto M, da Silva Herrero CFP, Pacola LM, Nunes AA, Defino HLA. Community Care Administration of Spinal Deformities in the Brazilian Public Health System. Clinics (São Paulo) 2017;72(08):485-490

3 Lima P Jr, Pellegrino L, Cafaro MF, Meves R, Landim E, Avanzi O. Escoliose idiopática do adolescente: perfil clínico e radiográfico da lista de espera para tratamento cirúrgico em hospital terciário de alta complexidade do Sistema Público de Saúde Brasileiro. Coluna/Columna 2011;10(02):111-115

4 Ahn H, Kreder H, Mahomed N, Beaton D, Wright JG. Empirically derived maximal acceptable wait time for surgery to treat adolescent idiopathic scoliosis. CMAJ 2011;183(09): E565-E570

5 Yang JH, Bhandarkar AW, Rathanvelu B, et al. Does delaying surgery in immature adolescent idiopathic scoliosis patients with progressive curve, lead to addition of fusion levels? Eur Spine J 2014;23(12):2672-2679

6 Dabke HV, Jones A, Ahuja S, Howes J, Davies PR. Should patients wait for scoliosis surgery? Orthop Proc 2006;88(Suppl II):225

7 Miyanji F, Newton PO, Samdani AF, et al. Impact of Surgical Waiting-List Times on Scoliosis Surgery: The Surgeon's Perspective. Spine 2015;40(11):823-828

8 Calman R, Smithers T, Rowan R. Impact of surgical waiting time on paediatric spinal deformity patients. ANZ J Surg 2013;83(12): 929-932

9 Miyanji F, Slobogean GP, Samdani AF, et al. Is larger scoliosis curve magnitude associated with increased perioperative health-care resource utilization?: a multicenter analysis of 325 adolescent idiopathic scoliosis curves J Bone Joint Surg Am 2012;94(09): 809-813

10 Clark S. Waiting times for scoliosis surgery. Lancet 2008;371 (9606):10-11

11 Tarrant RC, Queally JM, O'Loughlin PF, Sheeran P, Moore DP, Kiely PJ. Preoperative curves of greater magnitude $\left(>70^{\circ}\right)$ in adolescent idiopathic scoliosis are associated with increased surgical complexity, higher cost of surgical treatment and a delayed return to function. Ir J Med Sci 2016;185(02):463-471

12 Wright JG, Li K, Seguin C, et al. Development of pediatric wait time access targets. Can J Surg 2011;54(02):107-110

13 Bressan Neto M, Defino HLA. Surgical treatment of spinal deformities: clinical and epidemiological analisys of the consequences of underfunding [tese]. Ribeirão Preto: Universidade de São Paulo, Faculdade de Medicina de Ribeirão Preto; 2017
14 Asano LY, Filézio MR, Defino MP, Andrade VA, Cesar AE, Rodrigues LM. Radiographic Implications Of The Surgical Waiting List For The Treatment Of Spinal Deformity. Coluna/Columna 2018;17(01):19-22

15 Paim J, Travassos C, Almeida C, Bahia L, Macinko J. The Brazilian health system: history, advances, and challenges. Lancet 2011; 377(9779):1778-1797

16 Brasil. Lei ${ }^{\circ}$ 8.080, de 19 de setembro de 1990. Lei Orgânica da Saúde. Dispõe sobre as condições para a promoção, proteção e recuperação da saúde, a organização e o funcionamento dos serviços correspondentes e dá outras providências. Brasília, 1990. Disponível em: http://www.cofen.gov.br/lei-8080-lei-orgnica-da-saude_4163.html

17 Ferri-de-Barros F, Gibson J, Howard A. An argument for explicit rationing of health resources within the public-private mix in Brazil. Cad Saude Publica 2012;28(06):1211-1212

18 Ferri-de-Barros F, Andrew W, Howard AW, Martin DK. Inequitable Distribution Of Health Resources In Brazil: An Analysis of National Priority Setting. Acta Bioeth 2009;15(02):179-183

19 Inter-American Development Bank. Breve 16: Ethics of Health Resource Allocation in the Brazilian Publicly Financed Health Care System. Washington, DC: Inter-American Development Bank; 2016

20 Camarini PM, Rosanova GC, Gabriel BS, Gianini PE, Oliveira AS. The Brazilian version of the SRS-22r questionnaire for idiopathic scoliosis. Braz J Phys Ther 2013;17(05):494-505

21 Langensiepen S, Semler O, Sobottke R, et al. Measuring procedures to determine the Cobb angle in idiopathic scoliosis: a systematic review. Eur Spine J 2013;22(11):2360-2371

22 Gupta MC, Wijesekera S, Sossan A, et al. Reliability of radiographic parameters in neuromuscular scoliosis. Spine 2007;32(06): 691-695

23 Paixão ALSD. Reflections on the judicialization of the right to health and its implications in the SUS. Cien Saude Colet 2019;24 (06):2167-2172

24 Guimarães R. Technological incorporation in the Unified Health System (SUS): the problem and ensuing challenges. Cien Saude Colet 2014;19(12):4899-4908

25 Oudhoff JP, Timmermans DR, Knol DL, Bijnen AB, van der Wal G. Waiting for elective general surgery: impact on health related quality of life and psychosocial consequences. BMC Public Health 2007;7:164

26 Farley FA, Li Y, Jong N, et al. Congenital scoliosis SRS-22 outcomes in children treated with observation, surgery, and VEPTR. Spine 2014;39(22):1868-1874

27 McCall RE, Hayes B. Long-term outcome in neuromuscular scoliosis fused only to lumbar 5. Spine 2005;30(18):2056-2060

28 Ramo BA, Roberts DW, Tuason D, et al. Surgical site infections after posterior spinal fusion for neuromuscular scoliosis: a thirty-year experience at a single institution. J Bone Joint Surg Am 2014;96 (24):2038-2048

29 Basques BA, Chung SH, Lukasiewicz AM, et al. Predicting Shortterm Morbidity in Patients Undergoing Posterior Spinal Fusion for Neuromuscular Scoliosis. Spine 2015;40(24):1910-1917

30 Dayer R, Ouellet JA, Saran N. Pelvic fixation for neuromuscular scoliosis deformity correction. Curr Rev Musculoskelet Med 2012; 5(02):91-101

31 Martin CT, Pugely AJ, Gao Y, Weinstein SL. Causes and risk factors for 30-day unplanned readmissions after pediatric spinal deformity surgery. Spine 2015;40(04):238-246

32 Pitt DF, Noseworthy TW, Guilbert J, Williams JR. Waiting lists: management, legalities and ethics. Can J Surg 2003;46(03): 170-175

33 Abásolo I, Negrín-Hernández MA, Pinilla J. Equity in specialist waiting times by socioeconomic groups: evidence from Spain. Eur J Health Econ 2014;15(03):323-334 